\title{
Response of an ion-ion plasma to DC biased electrodes
}

\author{
Lara Popelier,* Ane Aanesland, and Pascal Chabert \\ Laboratoire de Physique des Plasmas, \\ Ecole Polytechnique, Palaiseau, France
}

(Dated: June 3, 2011)

\begin{abstract}
Electronegative plasmas are plasmas containing a significant fraction of negative ions, when magnetized they are very often segregated: the core is electropositive or weakly electronegative whereas a highly electronegative plasma forms at the periphery. At strong magnetic fields this segregation can lead to the formation of ion-ion plasmas almost free of electrons close to the walls or extraction surfaces and allows access to both positive and negative ions. The PEGASES thruster aims at alternately extract and accelerate positive and negative ions from the ion-ion plasma region to provide thrust by both types of ions. The acceleration schemes depends on the possible control of the potential in an ion-ion plasma relative to the acceleration grids. In this paper continuous extraction and acceleration of positive ions from the PEGASES thruster is investigated by a Retarding Field Energy Analyzer (RFEA). It is shown from the measured Ion Energy Distribution Functions (IEDF) that the continuous acceleration potential can be controlled by biasing bare electrodes in contact with the region of the plasma with high electron density (i.e. the weakly electronegative plasma core). A grounded grid placed in the ion-ion regions allowed consequently the acceleration of positive ions, where the ion velocity is controlled by the bias applied to the electrodes in the plasma core. On the contrary, when the grid in the ion-ion region is biased, positive ion beams were not detected downstream of the grid. The results indicate that biasing a grid positively in the ion-ion region results in an electronegative space charge sheath in front of the grid, which traps the positive ions inside the thruster.
\end{abstract}

PACS numbers:

${ }^{*}$ Electronic address: lara.popelier@lpp.polytechnique.fr 


\section{INTRODUCTION}

Classical electric propulsion systems provide thrust by the extraction and acceleration of positive ions from electropositive plasmas. In most cases Xenon is used due to its combination of low ionization potential and high atomic mass $[1,2]$. In a space environment, extracting and removing positive charges from the thruster induces a built up of negative polarization on the whole system [1]. An electron emitting hollow cathode placed at the thruster exit is added to neutralize the expelled ions, this produces a beam of zero net charge and avoids thrust cancellation. However, ion-electron recombination is a rather slow process and a plasma in the downstream region of the engine, the plasma plume, forms and may damage the spacecraft and in particular the cathode [2]. New concepts for electric propulsion are intensively studied in order to get rid of the neutralizer, and eventually to increase confidence in the electric propulsion system function and lifetime.

Ion-ion plasmas or electron-free plasmas can offer useful advantages in a variety of applications where neutral or quasi-neutral beams are used. Some examples are charge-free etching in the semiconductor industry [3, 4], neutral beam injections for fusion [5-7], or for electric propulsion in space applications [8]. Ideal ion-ion plasmas contain only positive and negative ions. In practice, a small amount of electrons may still be present, but in such highly electronegative plasmas the main negative charge carriers remain negative ions (on the contrary to the weakly electronegative plasmas where electrons are the main negative charge carrier). They can be obtained by two methods: i) by pulsing the plasma where the

ion-ion plasma appears as a transient region in the late afterglow [3] or ii) using a strong magnetic field segregating the plasma such that it appears as a separate region in the plasma $[9-11]$.

Knowledge of ion thruster principles and limitations together with a growing interest in ion-ion plasmas led to the development of the PEGASES ion thruster concept, meaning Plasma propulsion with Electronegative Gases [8, 12, 13]. An electronegative inductively coupled plasma is used as an ion source in which electrons are magnetically filtered so that an ion-ion plasma is obtained in the extraction stage. Positive and negative ions are then alternately accelerated by the means of biased grids to generate the thrust. Ion-ion recombination is often more efficient than electron-ion recombination [14, 15], so a beam with few charged particles in the downstream of the engine is expected and a neutralizing 
hollow cathode is no longer necessary.

The acceleration scheme currently being developed for the PEGASES thruster is based on "classical" gridded acceleration systems but adapted for alternate acceleration. How this will be done in practice depends on how ion-ion plasmas react or respond to biased grids or electrodes. The existing gridded thrusters (also called ion thrusters) have insulated walls allowing the plasma potential to float on top of the most positive potential surface in contact with the plasma. Hence, the plasma floats on top of the highly positively biased grid, the so-called plasma - or screen- grid which is in contact with the plasma. An electropositive space-charge sheath forms in front of the plasma grid, with thickness and magnitude of the potential drop determined by the electron temperature [16]. This operation, called plasma biasing, is not sufficient to generate an ion beam: a second grid, the acceleration grid, negatively biased with respect to the plasma grid, is needed to create the potential drop through which ions fall and are accelerated. More grids can be added to enhance beam directionality, ensure protection from backscattering, increase the lifetime of the grids, etc. $[1,2]$.

The ability to control the plasma biasing is in electropositive plasmas due to the electrons where an electropositive space charge sheath forms in front of all surfaces and the plasma is therefore always above the most positive potential [16]. Due to the lower temperature and greater mass of negative ions compared to electrons, the sheath structure in ion-ion plasmas changes from that of conventional electron-ion plasmas. Sheath formation and plasma biasing in ion-ion plasmas have been studied by numerical simulations [17, 18]. In these works pure ion-ion plasmas situated between two biased electrodes were investigated. It was shown that the plasma potential in the bulk is not the most positive potential of the system but sits halfway between the cathode and the anode potentials in the case of positive and negative ions of equal mass. The plasma potential gets slightly positive as the positive to negative mass ratio is increased. Draghici and Stamate [19] studied the possibility to tune the plasma potential in an ion-ion plasma by biasing a metallic cylinder in contact with the plasma in the ion-ion region. They demonstrated that the plasma potential (and hence the acceleration field or ion energy) does not change with the applied bias.

The purpose of this paper is to investigate further the possibility of plasma biasing of ionion plasmas in order to create beams with controllable energy/velocity. On the contrary to the above experiments and simulations, the biased electrodes are placed in the plasma core 
region, where the negative ion density is low and the plasma contains mobile high-energy

electrons. A single grid is placed in the extraction region (the ion-ion plasma region) to create a potential drop between the plasma and the downstream space, i.e. create a sheath, in which the ions are accelerated and eventually form an ion beam. Section II describes the experimental setup and diagnostic tools. Section III presents the different configurations studied in this paper. Results are presented and discussed in section IV and conclusions are given in section $\mathrm{V}$.

\section{EXPERIMENTAL SET-UP AND DIAGNOSTICS}

\section{A. The PEGASES prototype}

The cross section of the PEGASES thruster prototype is illustrated in figure 1 . The thruster body consists of a quartz cylinder $35 \mathrm{~cm}$ long and $6 \mathrm{~cm}$ diameter with two rectangular extractor tubes ( $4 \mathrm{~cm}$ by $4 \mathrm{~cm}$ and $6 \mathrm{~cm}$ long) attached perpendicular to the cylinder axis. The gas is introduced in the center of the cylinder. The plasma is created with a three-turn loop antenna wrapped around the middle of the quartz tube and continuously excited at $13.56 \mathrm{MHz}$ through a matching network using a $\pi$-circuit; the power supplied to the matching box is $300 \mathrm{~W}$ with less than $10 \mathrm{~W}$ reflected.

Four solenoides are placed symmetrically around the cylinder to create a $110 \mathrm{G}$ magnetic field with field lines parallel to the cylinder axis. A set of two neodymium magnets $20 \mathrm{~mm}$ wide, $40 \mathrm{~mm}$ long and $10 \mathrm{~mm}$ thick may be placed on each side of one extractor between $x=$ 45 and $65 \mathrm{~mm}$ to enhance the magnetic filtering in the extraction zone. Figure 2 shows the measured magnetic field strength as a function of position $x$ from the centre of the quartz cylinder through the middle of the extractor. The maximum magnetic field is about $800 \mathrm{G}$ located in the extractor.

Aluminum plates, with a surface of $16 \mathrm{~cm}^{2}$ each, terminate the cylinder ends and are perpendicular to the magnetic field lines. These endplates (also called electrodes) are in direct contact with the plasma and are either grounded or DC biased. The endplates bias, addressed as $V_{e p}$, is set between $-100 \mathrm{~V}$ and $+100 \mathrm{~V}$. One DC-biased grid can be placed in the extraction zone at $x=70 \mathrm{~mm}$. The grid made of aluminum is $1 \mathrm{~mm}$-thick with regularly spaced holes of $1 \mathrm{~mm}$-diameter, the surface is $13 \mathrm{~cm}^{2}$ with a $20 \%$ transparency. The grid is 
either grounded or bias between $-100 \mathrm{~V}$ and $+100 \mathrm{~V}$ (addressed as $V_{g}$ ). All biases applied to the thruster and diagnostics are referenced to a common ground, i.e. the vacuum chamber in which the thruster is placed.

The PEGASES thruster (volume $0.86 \mathrm{~L}$ ) is placed inside a large vacuum chamber, $1.2 \mathrm{~m}$ long and $0.6 \mathrm{~m}$ in diameter (volume $216 \mathrm{~L}$ ) and pumped via a turbomolecular pump and a primary dry pump (effective pumping system speed is around $2500 \mathrm{~L} \cdot \mathrm{s}^{-1}$ ) attached to a $30 \mathrm{~cm}$ port at the bottom of the large vacuum chamber. There are no available ports to measure the pressure inside the thruster itself $\left(P_{1}\right)$. The pressure is therefore monitored with a baratron (range $10^{-2}-10^{2}$ mTorr) placed in the large chamber, which pressure is addressed as $P_{2}$. The thruster volume is pumped through the two extractors and the pressure in the thruster $\left(P_{1}\right)$ can be estimated by [20]

$$
P_{1}=\frac{Q}{C}+P_{2}
$$

where $Q$ is the mass flow into the thruster and $C$ is the conductance of the two extractors.

For molecular flows $C=\frac{\Pi v_{t h}}{12} \frac{d^{3}}{l}$, where $v_{t h}$ is the thermal speed of the molecules and $d$ and $l$ are the diameter and length of the extractors (assuming cylindrical extractors for this estimate). In the experiments presented here, the $\mathrm{SF}_{6}$ mass flow is $28 \mathrm{sccm}$ (when operating without the grids), and $P_{2}$ is equal to 0.4 mTorr. The total conductance of the two extractors is $410 \mathrm{~L} . \mathrm{s}^{-1}$ which predicts an operating pressure inside the thruster of $1.3 \mathrm{mTorr}$. When the grid (20\% transparency) is inserted in one of the extractor, the conductance reduces to $15-20 \mathrm{~L} \cdot \mathrm{s}^{-1}$ and the estimated pressure for a flow between 14 and $28 \mathrm{sccm}$ is therefore 12-24 mTorr. In this estimate, outgassing is not included, which may further increase the pressure inside the thruster [20].

\section{B. Langmuir probe}

A Langmuir probe (LP) is used to measure current-voltage (IV) characteristics and thus provides information on whether an ion-ion plasma is formed in the extractor. The probe tip, $6 \mathrm{~mm}$ long and $0.25 \mathrm{~mm}$ diameter, is made of platinum to avoid etching by $\mathrm{SF}_{6}$. It is RF-compensated for the driving frequency $13.56 \mathrm{MHz}$ and its harmonic $27 \mathrm{MHz}$ with $\mathrm{RF}$ chokes [21]. The probe is introduced into the thruster via the extractor and allows spatially resolved measurements along the $x$-axis, i.e. perpendicularly to the magnetic field, except 
when the acceleration grid is introduced in the extractor as the probe can not pass through the grid.

In electronegative plasmas, the nature of the IV-characteristic depends on the electronegativity. For high electronegativity (large ratio of negative ion density to electron density) the IV-characteristic becomes symmetrical since the saturation currents (both at positive and negative polarisation of the probe) are mainly ion currents $[11,22]$. In the analysis carried out here, the negative and positive charge saturation currents are taken at the inflection points above and below the floating potential, respectively.

At the strongest magnetic field, in the intersection between the core and the extractor (Fig. 2), the electron Larmor radius $r_{L}$ is in the order of the probe radius when estimating the electron temperature to be from 1-2 eV in this region, and at the lower magnetic fields $r_{L}$ is orders or magnitude larger $\left(T_{e}\right.$ is about $6 \mathrm{eV}$ in the core at $\left.110 \mathrm{G}\right)$. Due to the large change in $r_{L}$ over the investigated region, the LP is not used to determine the plasma parameters from the IV-characteristics but is useful to indicate the existence of the ion-ion plasma.

\section{Retarding Field Energy Analyzer (RFEA)}

A Retarding Field Energy Analyzer (RFEA) is used to obtain the Ion Energy Distribution Functions (IEDFs). The analyzer designed in our laboratory has four plane grids and a collector plate with the total length of $1 \mathrm{~mm}$ [23]. All electrical components of the analyzer are made of stainless steel and the insulation material between the grids or plate is kapton. The grids wires are $11 \mu \mathrm{m}$ thick with grid holes of $40 \mu \mathrm{m}$, as a comparison the typical Debye length at the exit of the PEGASES thruster $\left(T_{e}=1-2 \mathrm{eV}, n=10^{10} \mathrm{~cm}^{-3}\right)$ is $75-100 \mu \mathrm{m}$ which is acceptable for good IEDF measurements [23]. Each grid has a $60 \%$ transparency to reduce both the ion density and the neutral density. The entrance grid $\mathrm{G}$, facing the plasma, is fixed on a grounded $50 \mathrm{~mm}$-diameter diaphragm with an aperture $3 \mathrm{~mm}$ in diameter to reduce the open area seen by the plasma. The analyzer is differentially pumped to reduce the effect of collisions within the analyzer. The external dimensions of the RFEA are larger than the extractor, all measurements with the analyzer are therefore obtained at $x=82 \mathrm{~mm}$ and cannot be done in the extractor.

The voltages applied to the analyzer grids are shown in figure 3. The aperture grid $\mathrm{G}$ potential is grounded, the second repeller grid $\mathrm{R}$, is negatively biased to $-30 \mathrm{~V}$, the 


\begin{tabular}{|c|c|c|c|c|}
\hline Configuration & $\mathrm{A}$ & $\mathrm{B}$ & $\mathrm{C}$ & $\mathrm{D}$ \\
\hline \hline Endplates & biased & biased & grounded & biased \\
\hline Grid & none & grounded & biased & grounded \\
\hline Magnetic field & strong & weak & weak & strong \\
\hline Flow rate (sccm) & 28 & 28 & 28 & 14 \\
\hline$P_{1}$ (mTorr) & 1.3 & 24 & 24 & 12 \\
\hline$P_{2}$ (mTorr) & 0.4 & 0.4 & 0.4 & 0.3 \\
\hline
\end{tabular}

TABLE I: Overview of the various configurations.

discriminator grid D is swept to discriminate the ion kinetic energies. A fourth grid, the secondary grid $\mathrm{S}$, is introduced to recuse the effect of secondary electrons created from grid $\mathrm{D}$ and from the collector $\mathrm{C}$, and is biased to $-40 \mathrm{~V}$. The collector $\mathrm{C}$ measures the ion current and is held positive compared to $\mathrm{S}$, at $-35 \mathrm{~V}$. The voltage supply and data acquisition are obtained by the Semion RFEA system from Impedans [24].

The IEDF is proportional to the derivative of the IV-characteristic and the area under the curve of the derivative is proportional to the ion density. When the potential in the plasma is isotropic, i.e. no internal structures and the mean free path is larger than the system length, the IEDF is a narrow gaussian distribution and shows the energy of the ions falling through the sheath in front of the analyzer. The plasma potential is then deduced from the voltage at the maximum of the IEDF and addressed as $V_{p}$.

\section{DIFFERENT CONFIGURATIONS STUDIED}

In order to investigate the acceleration scheme in the PEGASES thruster, four experimental configurations are studied and represented on figure 4. In the intention to simplify the diagrams, only one extractor is shown. The illustrations show the endplates and grid bias scheme and the applied magnetic fields. An overview of the different configurations are given in table I. The pressures inside the thruster core $P_{1}$ and in the larger chamber $P_{2}$ are affected by the presence of the grid (as discussed and calculated in section II A), and their values are given in table I. In all these configurations the grounded RFEA is placed $1 \mathrm{~cm}$ from the extractor end (except when the LP is used). 
- Configuration A The purpose of this configuration is to study the plasma biasing in the case of a classical electropositive plasma and in the case of an ion-ion plasma, Argon and $\mathrm{SF}_{6}$ are therefore used in this configuration. The endplates are biased from $-40 \mathrm{~V}$ to $+40 \mathrm{~V}$. There is no grid in the extractor to allow LP probe measurements inside the thruster. The magnets arranged along the extractor are used to strengthen the magnetic field and are required to form an ion-ion plasma in the extractor [13].

- Configuration B As for configuration A, the endplates are DC biased, but in addition a grounded grid is inserted in the extractor in order to form an ion beam downstream of the grid. In this first study we use Argon and since the grid has only a $20 \%$ transparency the magnets had to be removed to allow higher plasma density in the extractor and therefore a better signal-to-noise ratio for the RFEA. The magnetic field is therefore generated only by the solenoides. The flow rate was the same as in configuration A and the pressure in the thruster is now about 24 mTorr.

- Configuration C This configuration is equivalent to B, except for the endplates which are grounded and the grid which is DC biased. The purpose is here to compare the results between biasing the endplates $(\mathrm{B})$ and biasing the grid in the extractor $(\mathrm{C})$. Again Argon is used with the same flow rate as in cases A and B, yielding a pressure of 24 mTorr in the thruster, and the weak magnetic field is applied.

- Configuration D In order to study the positive ion beam formation in the case of an ion-ion plasma, the endplates are DC biased whereas the grid is grounded. The strong magnetic field is used to generate the ion-ion plasma (as in Configuration A). The flow rate is reduced from $28 \mathrm{sccm}$ (used in the three other cases) to $14 \mathrm{sccm}$, and the pressure inside the thruster is estimated to be around 10-12 mTorr. This reduced pressure is necessary to increase the signal-to-noise ratio in the RFEA.

- Configuration $\mathbf{E}$ This configuration is not shown in figure 4. It is equivalent to D with a strong magnetic field to generate an ion-ion plasma, but now the endplates are grounded and the grid is biased such as in configuration C. The purpose is again to study the difference between biasing the endplates (D) and the grid (E). 


\section{RESULTS AND DISCUSSION}

\section{A. Configuration A, Plasma biasing}

In this subsection configuration $\mathrm{A}$ is used, with the endplates biased and a strong magnetic field, applied to both Argon and $\mathrm{SF}_{6}$ plasmas.

Figure 5 shows the positive and negative saturation currents measured by the LP as a function of position from the cylinder axis towards the exit of the extractors in the $\mathrm{SF}_{6}$ plasma. The region inside the cylinder (in the thruster core) is weakly electronegative with a large electron current, while in the extraction region the plasma is strongly electronegative with equal magnitudes for the positive and negative currents. The inset shows two Langmuir probe characteristics in logarithmic scale measured at $x=1 \mathrm{~mm}$ and $x=80 \mathrm{~mm}$ showing non-symmetrical and symmetrical characteristics, respectively. The figure indicates that the plasma in the cylinder or thruster core is dominated by the electron dynamics, while the plasma in the extractors is dominated by the positive and negative ion dynamics.

Figures 6 and 7 show IEDFs at $x=82 \mathrm{~mm}$ for various biases on the cylinder endplates in the case of Argon and $\mathrm{SF}_{6}$, respectively. In both plasmas, the IEDFs are narrow gaussian distributions where the center voltage $V_{p}$ increases with $V_{e p}$ for positive biases, while for negative biases, $V_{p}$ saturates around $15 \mathrm{~V}$. The deduced plasma potential (from fig. 6 and 7 ) is plotted as a function of the endplates bias in figure 8 , and shows that $V_{p}$ increases linearly with $V_{e p}$ for positive voltages and can be expressed as

$$
V_{p}=V_{p 0}+V_{e p}
$$

where $V_{p 0}$ is the plasma potential when the endplates are grounded.

It can be seen from figure 6 and 7 that the height of the IEDF decreases with $V_{e p}$. From the corresponding IV-characteristics, the total current measured at zero voltage on the discriminator grid is constant, indicating a constant ion flux with increasing $V_{e p}$. Hence, as the ion velocity is increased, the outgoing density is decreased.

Figure 9 shows the Langmuir characteristics obtained in $\mathrm{SF}_{6}$ at $x=60 \mathrm{~mm}$, i.e. in the ion-ion region, for endplate biases of $-40 \mathrm{~V}, 0 \mathrm{~V}$ and $+40 \mathrm{~V}$. The characteristics are symmetrical for each value of $V_{e p}$. Biasing the endplates does not affect the shape of the curve (i.e. the ion-ion plasma formation) but the floating potential follows $V_{e p}$.

The electrons are very mobile along the cylinder axis (parallel to the magnetic fieldlines) 
and dominate the plasma equilibrium and the sheath formation in this region [25, 26]. Consequently, classical electropositive sheaths form in front of the grounded or biased endplates with thickness and potential drop given by the electron temperature in this region. This temperature is most likely not affected by the endplates bias $V_{e p}$ since the power and the pressure are constant. The plasma potential $V_{p 0}$ is therefore constant and sits on top of the biased endplates voltage and the potential in the thruster core is therefore given by Eq. 2 (as seen both by the RFEA and the LP).

There needs to be a high plasma conductivity for this potential to be isotropic along the $x$-axis, i.e. perpendicular to the magnetic field. Since the electrons mobility is significantly reduced by the strong magnetic field, we expected a reduced plasma conductivity and a potential drop in the magnetic filter region. However, the single peaked IEDFs and the floating potential measured by the IV-characteristics in the ion-ion plasma suggest a good conductivity between the core and the extractor and a rather flat plasma potential along the $x$-axis.

The plasma potential saturates at $15 \mathrm{~V}$ when the endplates are biased negatively compared to the grounded RFEA. This is because $V_{e p}$ is no longer the most positive potential in the system: the large body of the RFEA introduces ground and the ions are accelerated in the sheath created in front of it. It is interesting to note however that the $15 \mathrm{~V}$ saturation correspond to a typical sheath potential of electropositive plasmas with an electron temperature of about $3 \mathrm{eV}$, and this is obtained also in the case of $\mathrm{SF}_{6}$ when the ion-ion plasma is in front of the analyzer.

\section{B. Configuration B, Positive ion beam formation in Argon with biased endplates}

In the following section a grounded grid is placed in the extractor. The measured IEDFs give evidence of positive ion beams downstream of the grid, and the mean ion energy is now adressed as the beam potential $V_{b}$ instead of $V_{p}$ in the previous configuration.

Figure 10 shows IEDFs obtained in Argon for various applied voltages on the endplates. The center voltage of the IEDF, $V_{b}$, is increased with $V_{e p}$ but surprisingly much less than in configuration A. Figure 13 shows $V_{b}$ as a function of the endplates bias (open squares), and shows that the increase in $V_{b}$ is not a linear function with $V_{e p}$ as in the previous case (points at $V_{e p}=20 \mathrm{~V}$ and $60 \mathrm{~V}$ were obtained with two grounded grids in the extractor, 
separated by $4 \mathrm{~mm}$, with consistent results when using one or two grounded grids). The beam potential can now be expressed as

$$
V_{b}=V_{p 0}+V_{e p}-\Delta V
$$

where $\Delta V$ is for now a "loss term". Its physical meaning is discussed below.

Similar to configuration A, the sheath in front of the endplates will be a low potential sheath given by $T_{e}$ in the thruster core and the plasma in this region will float on top of the endplates bias. If the conductivity in the plasma is high, which should be the case with this weak magnetic field (and also indicated by the previous results), one would expect a uniform potential along $x$ and the potential corresponding to $V_{p 0}+V_{e p}$ (equivalent to Eq. 2) should drop in the sheath in front of the grid. The ions falling through this high-voltage sheath should be accelerated and measured downstream of the grid with a corresponding beam energy. Figure 13 shows that the ions do not acquire this energy, but loose some energy $\Delta V$ "along the way".

One possible explanation for this energy loss is as follows. Due to the low transparency of the grids, the pressure in the thruster volume $P_{1}$ is increased from about $1.3 \mathrm{mTorr}$ (without the grid) to about 24 mTorr (with the grid) using the same flow rate (see section II A). The ion mean free path $\lambda_{i}$ changes consequently from $2.3 \mathrm{~cm}$ to $0.12 \mathrm{~cm}$ at this high pressure and is therefore much smaller than the system length [27]. If there is a potential decrease $\Delta V$ from the core towards the grid, the ions will lose by charge exchange collisions the corresponding energy that they would gain in this field otherwise. The potential drop in the sheath in front of the grid is then not given by $V_{p 0}+V_{e p}$, but reduced by the voltage decrease $\Delta V$ along $x$. The beam energy measured downstream of the grid would in this case correspond to the energy the ions acquire crossing the sheath in front of the grid and will be given by eq. 3 .

In such a collisional case one would normally expect a broadening of the IEDF towards lower energies (slightly observed in the IEDF at $V_{e p}=0 \mathrm{~V}$ ). The narrow distribution is probably due to the local ionization ensuring that all the ions undergo many collisions.

The loss term $\Delta V$ introduced in equation 3 can therefore be understood as a potential decrease between the source and the grid. $\Delta V$ is plotted (squares) as a function of endplates bias in figure 14 showing that the "loss term" (or potential decrease) increases with increasing endplates bias and saturates at about $20 \mathrm{~V}$. 


\section{Configuration C, Positive ion beam formation in Argon with biased grid}

Figure 11 shows IEDFs in case $\mathrm{C}$ when the grid is biased and the endplates are grounded. Note that, since the grid and the RFEA are at different potentials, the ions may be accelerated in the electric field between the grid and the grounded entrance of the RFEA. For grid potentials between $-40 \mathrm{~V}$ and $40 \mathrm{~V}$ the IEDFs are relatively noisy, the peak amplitude is low and the beam potential is constant at around $17 \mathrm{~V}\left(V_{b 0}\right)$. For grid biases from $60 \mathrm{~V}$ and above, the IEDFs are more pronounced with a single peak, which amplitude is high and decreasing with increasing applied voltage, so that the beam potential increases linearly with the applied voltage on the grid (i.e. an increment of $10 \mathrm{~V}$ in $V_{g}$ results in an increase of $10 \mathrm{~V}$ in $\left.V_{b}\right) . V_{b}$ is plotted as a function of the grid bias in figure 12 (triangles) and can be expressed as a stepwise function given by

$$
V_{b}= \begin{cases}V_{b 0} & \text { when } V_{g}<50 \mathrm{~V} \\ V_{b 0}+V_{g}-50 & \text { when } V_{g} \geq 50 \mathrm{~V} .\end{cases}
$$

This result may be understood by comparing the sheath thickness in front of the grid with the diameter of the grid holes. In the case of a Child-Langmuir sheath, for a given current extracted from the plasma the sheath thickness increases as the $3 / 4$ power of the voltage across the sheath [27]. For a $15 \mathrm{~V}$ potential drop the sheath thickness is $s \simeq 4 \lambda_{D e} \simeq 0.4 \mathrm{~mm}$, while for a $50 \mathrm{~V}$ potential drop $s \simeq 10 \lambda_{D e} \simeq 1 \mathrm{~mm}\left(\right.$ for $n=10^{10} \mathrm{~cm}^{-3}$ and $\left.T_{e}=2 \mathrm{eV}\right)$. These values are calculated for a collisionless case and are slightly modified for intermediate pressures [27]. The diameter of the grid holes are $1 \mathrm{~mm}$, which indicates that at low $V_{g}$, the grid is transparent to the plasma which is able to pass through the grid without being accelerated. The measured IEDF corresponds to the low density plasma in front of the RFEA with the plasma potential corresponding to $V_{b 0}$.

Above 50 volts, the sheath thickness in front of the grid may become larger than the radius of grid apertures. The potential difference between the plasma and the grid could therefore be equal to $V_{b 0}$, and one would then expect a positive ion beam from the plasma towards the analyzer with an energy corresponding to the sum of the potential drop in the sheath in front of the grid and in the electric field between the grid and the RFEA, i.e. $V_{b 0}+V_{g 1}$. However, note that the beam energy is $50 \mathrm{~V}$ less than expected when the grid is biased above $50 \mathrm{~V}$, which cannot be explained by the above scenario.

Another hypothesis is the possible formation of an anodic sheath (also described as a 
field reversal) in front of the grid [28]. The electrons of the upstream plasma (the main plasma produced in the thruster chamber) may be extracted and accelerated across this anodic sheath and strike a plasma between the grid and the analyzer. The potential of this downstream plasma would be controlled by, but somewhat smaller than, the grid potential, because of the potential drop in the anodic sheath. Positive ions generated in the downstream plasma would be accelerated in the positive space charge sheath formed in front of the analyzer and detected with a potential below the grid potential.

\section{Configuration D, Positive ion beam formation in $\mathbf{S F}_{6}$ with biased endplates}

Figure 12 shows the IEDFs obtained in configuration D using $\mathrm{SF}_{6}$ with a flow rate of 14 sccm. In this case an ion-ion plasma is formed in the extractors. As in configuration B, the beam potential $V_{b}$ increases with $V_{e p}$ and the beam energy can be expressed similar to equation 3. $V_{b}$ and the loss term $\Delta V$ are plotted (diamonds) as a function of the endplates bias in figure 13 and 14, respectively. No signal were measured with the RFEA for negative endplates biases. Compared to the IEDFs obtained in Argon, these distributions have a significant signal-to-noise ratio mainly due to much lower currents collected by the RFEA. At endplate biases above $80 \mathrm{~V}$, a broadening towards lower energies is observed due to charge exchange collisions.

Both the ion velocity, $v=\sqrt{2 e V_{b} / m_{i}}$ (where $m_{i}$ is the ion mass), and ion density, given by the integral of the IEDF, increases as a function of $V_{e p}$. Hence the outgoing ion flux increases with the endplates bias. This is not the case in configuration A without the grid, where the ion flux is constant as a function of $V_{e p}$.

\section{E. Configuration E, Positive ion beam formation in $\mathbf{S F}_{6}$ with biased grid}

The measured current to the RFEA was negligible in the case of an ion-ion plasma in front of the grid, when a positive bias was applied to the grid and the endplates were grounded (similar to configuration C). This indicates that in the case of an ion-ion plasma, a negative space charge sheath may form in front of the positively biased grid and thus prevents

positive ions to exit the thruster. This result is in agreement with previous experimental and modeling works [17-19] 


\section{CONCLUSION}

The PEGASES thruster aims at alternately extract and accelerate positive and negative ions from an ion-ion plasma region in a magnetized highly electronegative plasma. This acceleration scheme will provide thrust without requiring an additional neutralization source. The acceleration scheme depends on the possibility to control the potential in an ion-ion plasma, relative to the acceleration grid. Previous works from Draghici and Stamate [19] showed that the plasma potential in an ion-ion plasma, isolated from ground, does not change with biases applied to electrodes in contact with the ion-ion plasma. The purpose of this paper was to investigate further the possibility of plasma biasing of the ion-ion plasma in order to control the mean velocity of the created ion beams. The electronegative plasma studied here was highly segregated with a core comprising mainly positive ions and electrons, and an extraction region comprising mainly positive and negative ions. The biasing scheme or the acceleration scheme was based on placing one electrode in contact with the plasma in the region of high electron density (here endplates in the thruster core) and placing one grid in the ion-ion region (extraction region). Different scenarios were investigated, such as biasing the endplates while the grid was grounded, and inverse biasing the grid while the electrodes were grounded.

The results presented here showed that biasing the electrodes situated in the electropositive core allows to control the potential of the ion-ion plasma. When the grid placed in the ion-ion region was grounded (while biasing the endplates), the ions were accelerated in the sheath in front of this grid and the acceleration potential then depended on the endplates bias. Hence, ion beams with controllable energy could be obtained downstream of the grounded grid. We first expected the beam potential to be equal to the sum of the potential applied to the electrodes and the sheath potential of a Child-Langmuir sheath, however collisions inside the thruster could be responsible for the lower beam potential measured downstream of the grid.

In the case when the grid in the ion-ion plasma region was biased, no downstream ion beam were measured. This indicated that when the grid is biased positively in the ion-ion

plasma region, a negative space charge sheath formed in front of this grid. This sheath prevented positive ions to exit the thruster through the grid and no ion beam were formed. Those results are in agreement with the experimental work by Draghici and Stamate [19] 
and the simulations by Midha and Economou [17] and Meige et al. [18].

\section{Acknowledgment}

This work is supported by EADS-Astrium.

[1] R.G. Jahn, Physics of Electric Propulsion, New York: McGraw-Hill, chapter 7 (1968).

[2] D.M. Goebel, I. Katz, Fundamentals of Electric Propulsion: Ion and Hall Thrusters, John Wiley \& Sons (2008).

[3] D.J. Economou, Fundamuntals and applications of ion-ion plasmas, Appl. Surf. Sci. 253, 6672 (2007).

[4] S. Samukawa, K. Sakamoto, K. Ichiki, Generating high-efficiency neutral beams by using negative ions in an inductively coupled plasma source, J. Vac. Sci. Technol. A, 20, 1566 (2002).

[5] U. Fantz, Basics of plasma spectroscopy, Plasma Sources Sci. Technol. 15, S137 (2006).

[6] L. Grisham, J. Kwan, Perspective on the role of negative ions and ion-ion plasmas in heavy ion fusion science, magnetic fusion energy, and related fields, Nucl. Instrum. Meth. Phys. Res. A 606, $83(2009)$.

[7] L. Grisham, J.W. Kwan, S.K. Hahto, K.N. Leung, G. Westenskow, Negative halogen ions for fusion applications, Rev. Sci. Instrum. 77, 03 A501 (2006).

[8] A. Aanesland, A. Meige, P. Chabert, Electric propulsion using ion-ion plasmas, Journal of Physics: Conference Series 162, 012009 (2009).

[9] K.N. Leung, K.W. Ehlers, M. Bacal, Extraction of volume produced H-ions from a multiscup source, Rev. Sci. Instrum. 54,56 (1983).

[10] E. Stamate et K. Ohe, Determination of negative-ion and electron parameters in an Ar/SF6 plasma, J. Appl. Phys. 84, 2450 (1998).

[11] P. Chabert, T.E. Sheridan, R.W. Boswell, J. Perrin, Electrostatic probe measurement of the negative ion fraction in an $S F_{6}$ helicon discharge. Plasma Sources Sci. Technol. 8, 561 (1999).

[12] P. Chabert, WO 2007/065915 A1 (2007).

[13] A. Aanesland, G. Leray, P. Chabert, Pegases - Plasma propulsion with electronegative gases, AIAA 2008-5185 (2008). 
[14] P.N. Barnes, M.J. Kushner, Ion-ion neutralization of iodine in radio-frequency inductive discharges of Xe and I2 mixtures, J. Appl. Phys. 82, 2150 (1997).

[15] I.M. Littlewood, Ion-ion recombination in the tertiary mixtures CH4:SF6:He and CH4:SF6:Ar, J. Phys. D: Appl. Phys. 23, 308 (1990).

[16] M.A. Lieberman, A.J. Lichtenberg, Principles of Radiofrequency Discharges and Material Processing, 2nd edition, New York: Wiley-Interscience (2005).

[17] V. Midha, D.J. Economou, Dynamics of ion-ion plasmas under radio frequency bias, J. Appl. Phys. 90, 1102 (2001).

[18] A. Meige, G. Leray, J.L. Raimbault, P. Chabert, Appl. Phys. Lett. 92, 061501 (2008).

[19] M.Draghici, E. Stamate, Properties of highly electronegative plasmas produced in a multipolar magnetic-confined device with a transversal magnetic filter, J. Phys. D: Appl. Phys. 43 (2010).

[20] J.F. O'Hanlon, A Users Guide to Vacuum Technology, 2nd Edition, John Wiley and Sons, New York (1989).

[21] R.R.J. Gagné, A. Cantin, Investigation of an rf plasma with symmetrical and asymmetrical electrostatic probes, J. Appl. Phys. 43, 6 (1972).

[22] G.A. Woolsey, I.C. Plumb, D.B. Lewis, Langmuir probe characteristics in a positiveion/negative-ion plasma, J. Phys. D: Appl. Phys. 6, 1883 (1973).

[23] A. Perret, P. Chabert, J. Jolly, J.-P. Booth, Ion ernergy unifomity in high-frequency capacitive discharges, Appl. Phys. Lett. 86, 021501 (2005).

[24] D. Gahan, B. Dolinaj, M.B. Hopkins, Retarding field analyzer for ion energy distribution measurements at a radio-frequency biased electrode, Rev. Sci. Instrum. 79, 33502 (2008)

[25] N.S.J. Braithwaite, J.E. Allen, Boundaries and probes in electronegative plasmas, J. Phys. D: Appl. Phys. 21, 1733 (1988).

[26] G. Leray, P. Chabert, A.J. Lichtenberg, M.A. Lieberman, Fluid model of an electronegative discharge with magnetized electrons and unmagnetized ions, J. Phys. D: Appl. Phys. 42, 4020 (2009).

[27] P. Chabert, N.S.J. Braithwaite, Physics of Radio-Frequency Plasmas, Cambridge University Press (2011).

[28] L. Conde, C. Ferro Fontan, J. Lambas, The transition from an ionizing electron collecting plasma sheath into an anodic double layer as a bifurcation, Phys. Plasmas 13, 113504 (2006). 


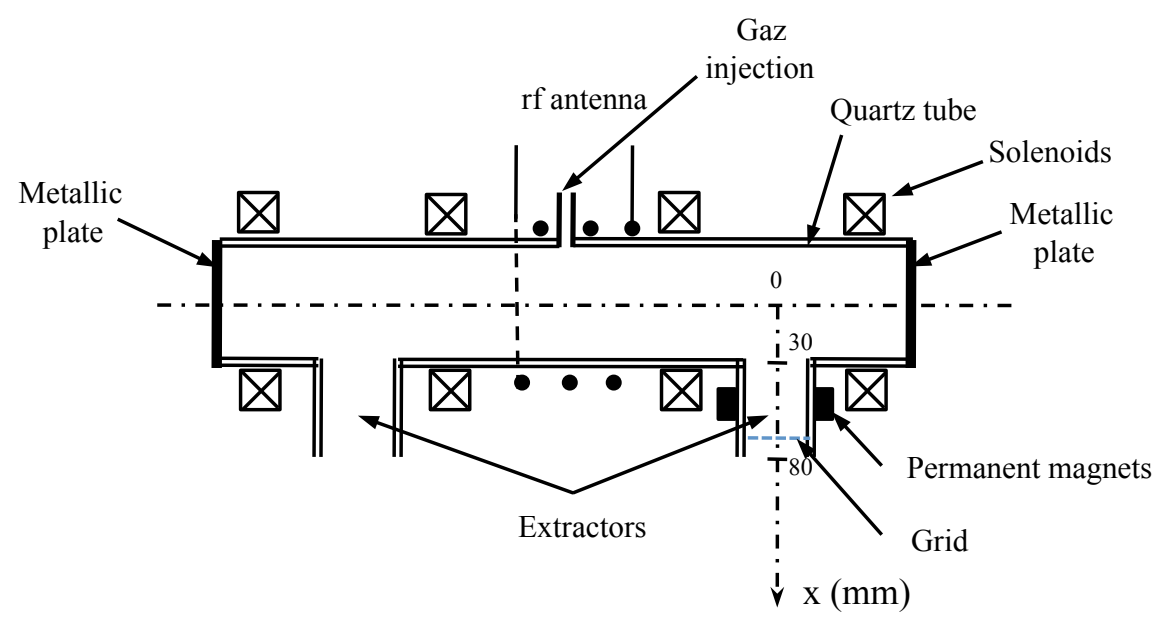

FIG. 1: The cross section of the first PEGASES thruster prototype.

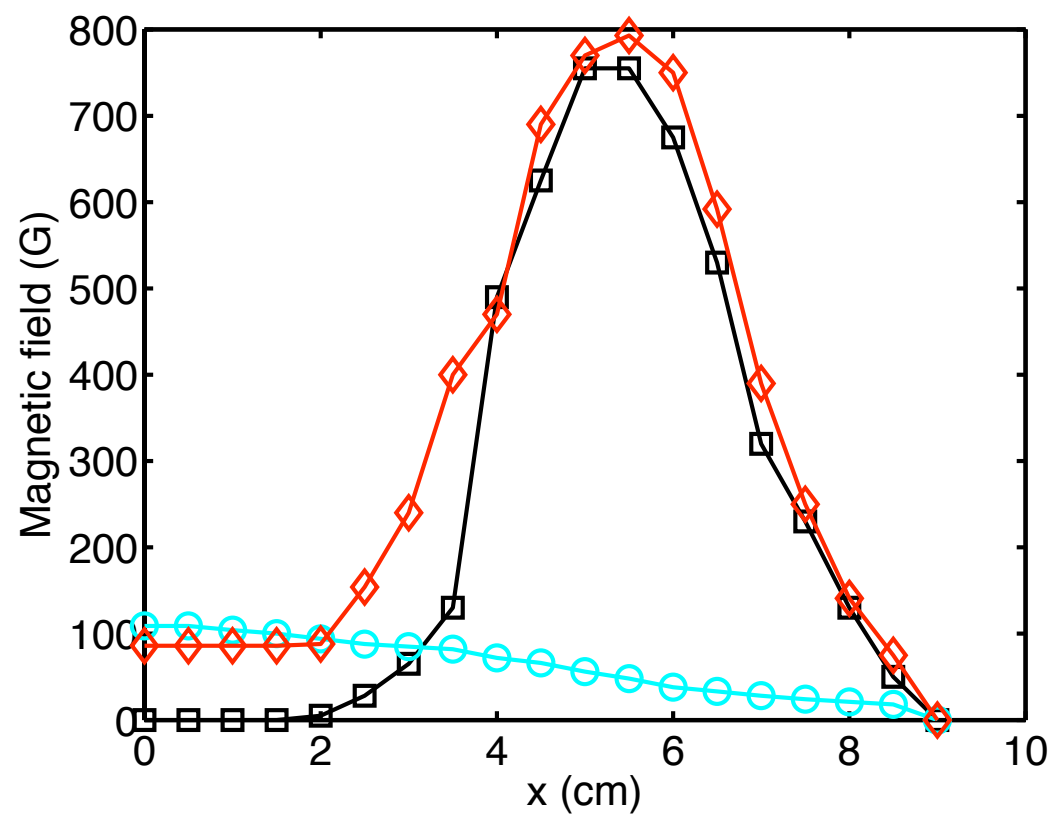

FIG. 2: The magnetic field strength as a function of position $x$ from the cylinder axis through the middle of the extractor. Blue circles are obtained by the solenoides only, black squares by the permanent magnets and red diamonds by both the solenoides and magnets. 


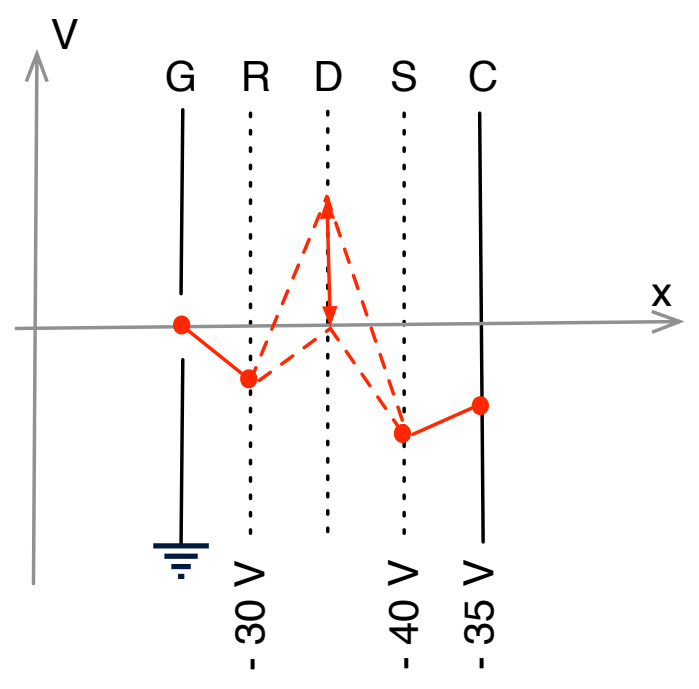

FIG. 3: Schematic of the grid biases in the RFEA, where the discriminator grid is swept over the ion energy range. $\mathrm{G}$ stands for grounded grid, $\mathrm{R}$ for repeller grid, D for discriminator grid, $\mathrm{S}$ for secondary grid, C for collector plate. 

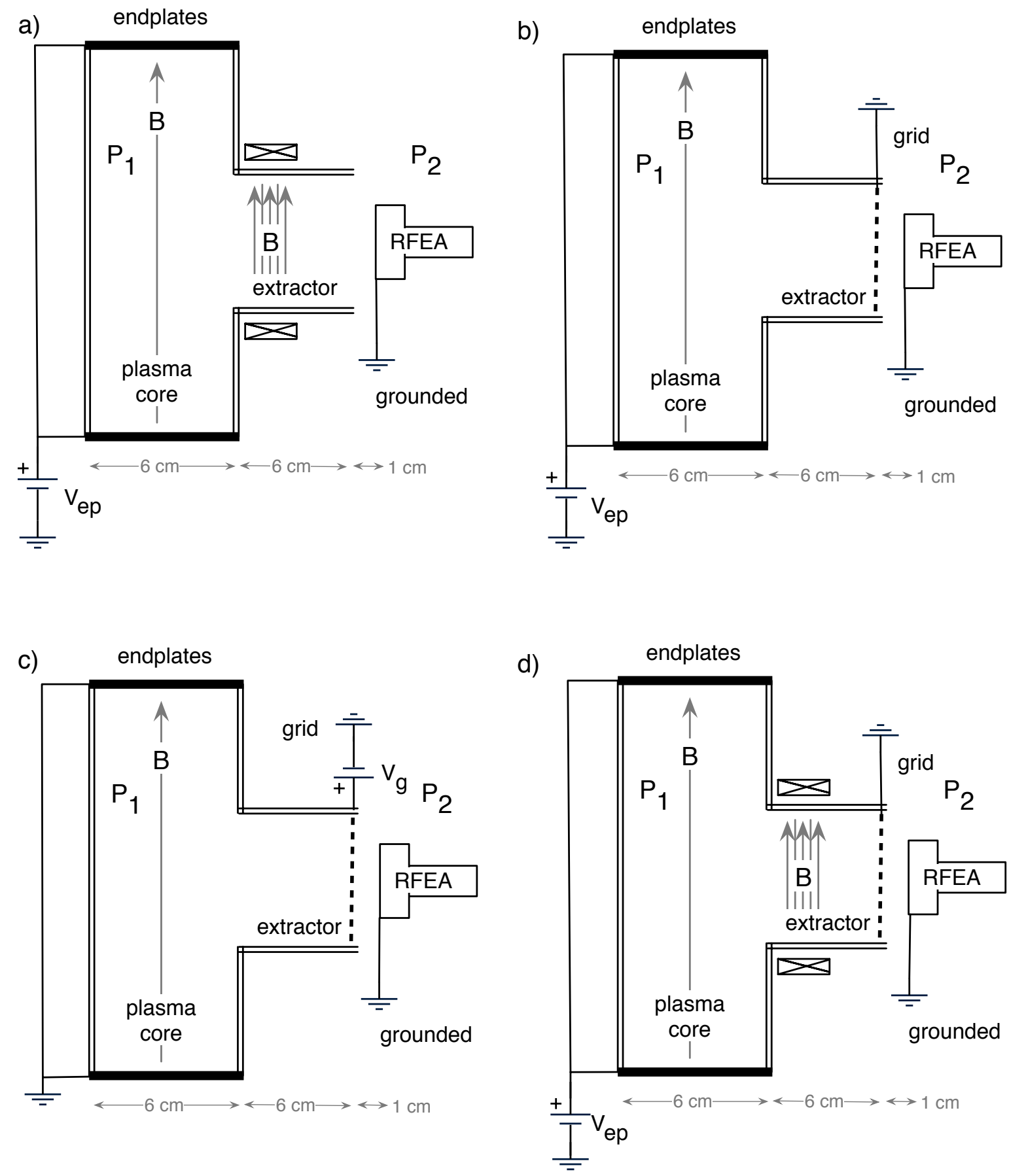

FIG. 4: Illustration of the four configurations studied in this paper. a) A is used for both Argon and $\mathrm{SF}_{6}, \mathrm{~B}$ and $\mathrm{C}$ only with Argon, $\mathrm{D}$ with $\mathrm{SF}_{6}$ on ly. 


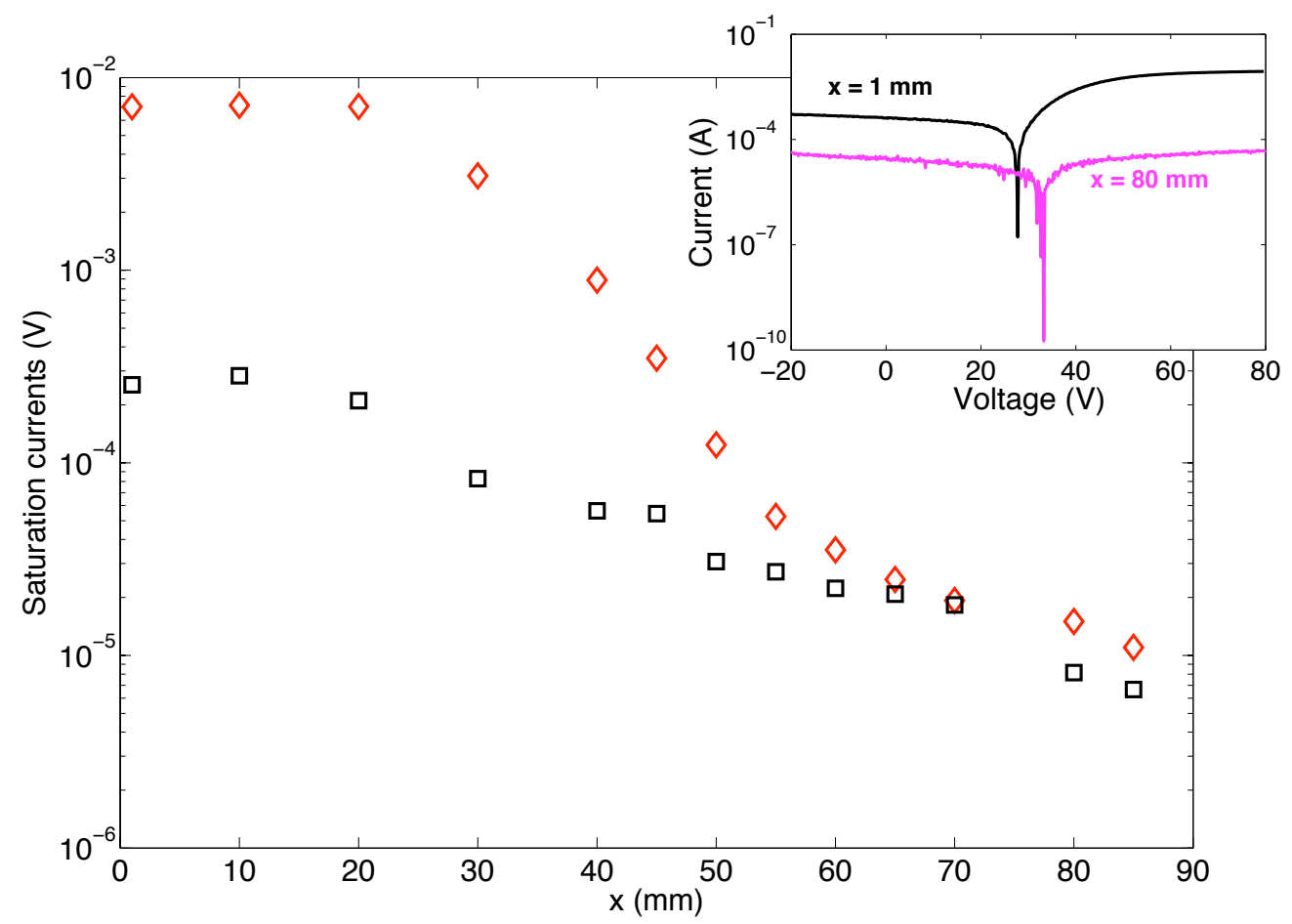

FIG. 5: Positive (red diamonds) and negative (black squares) saturation currents as a function of position $x$ from the centre of the thruster through the extractor measured with the LP. The $\mathrm{SF}_{6}$ plasma is generated with a gas flow of $28 \mathrm{sccm}$ and configuration $\mathrm{A}$ is used. The inset represents IV-characteristics in logarithmic scale for $x=1 \mathrm{~mm}$ and $80 \mathrm{~mm}$. 


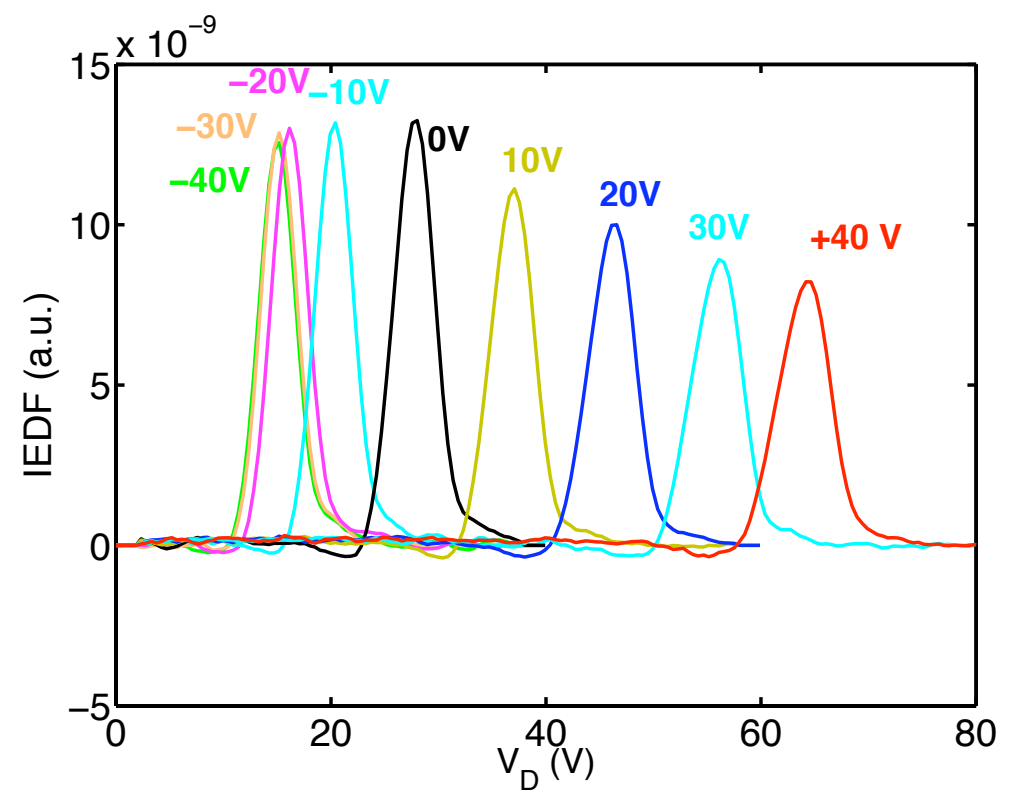

FIG. 6: IEDFs for positive ions in Argon obtained with configuration A for various endplates biases from $-40 \mathrm{~V}$ to $+40 \mathrm{~V}$ ( $V_{e p}$ indicated on each IEDF).

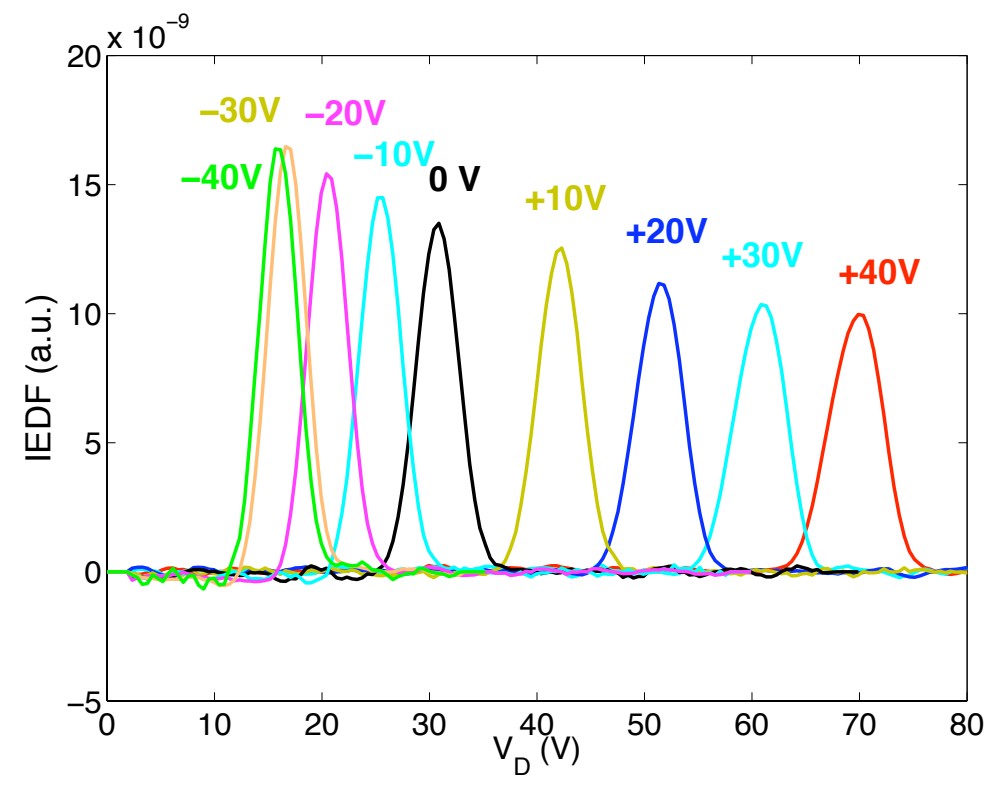

FIG. 7: IEDFs for positive ions in $\mathrm{SF}_{6}$ obtained with configuration A for various endplates biases from $-40 \mathrm{~V}$ to $+40 \mathrm{~V}$ ( $V_{e p}$ indicated on each IEDF). 


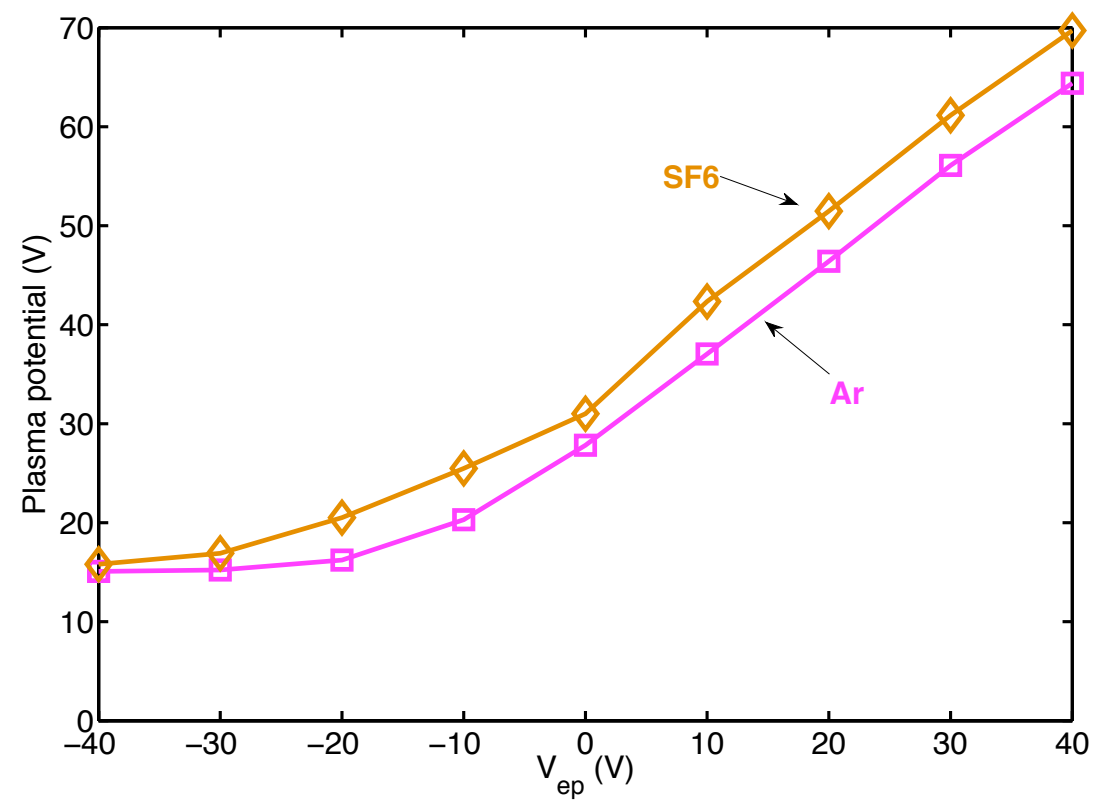

FIG. 8: Plasma potential $V_{p}$ as a function of endplates bias $V_{e p}$ obtained from the IEDFs shown in figure 6 (squares) and figure 7 (diamonds).

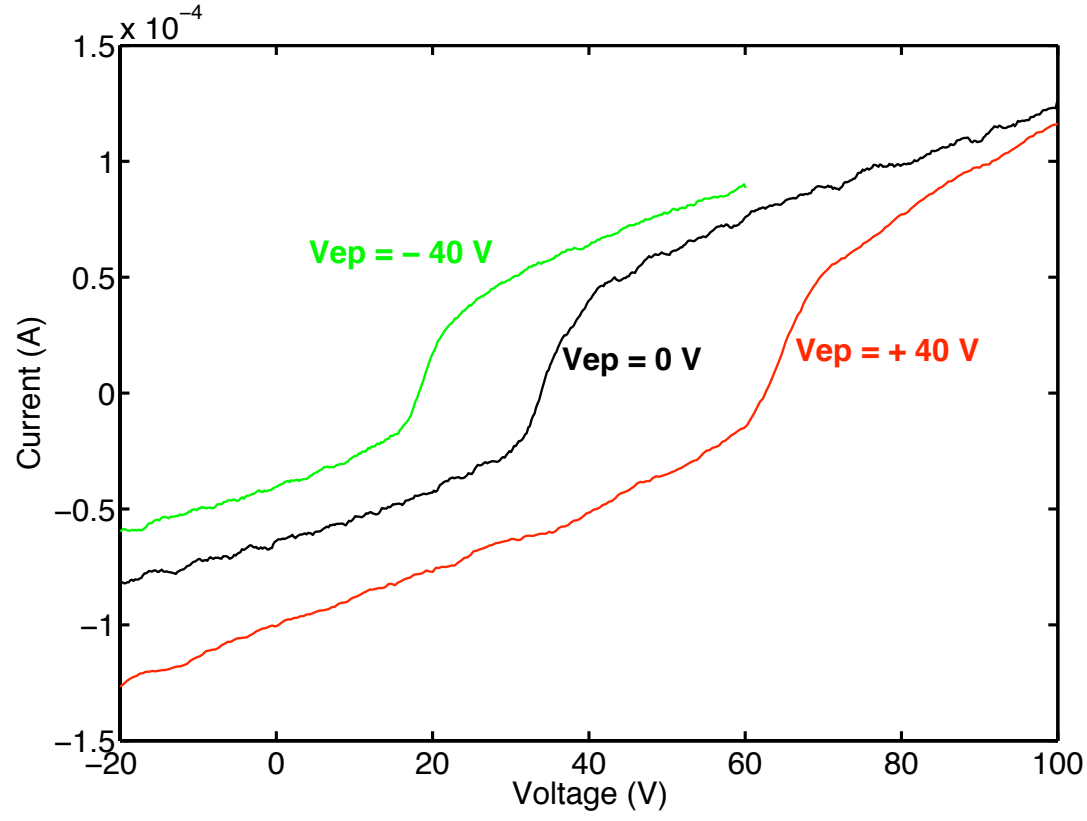

FIG. 9: Langmuir probe IV-characteristics obtained at $x=60 \mathrm{~mm}$ for endplates bias equal to $-40 \mathrm{~V}, 0 \mathrm{~V}$ or $+40 \mathrm{~V}$. Configuration $\mathrm{A}$ is used with $\mathrm{SF}_{6}$. 


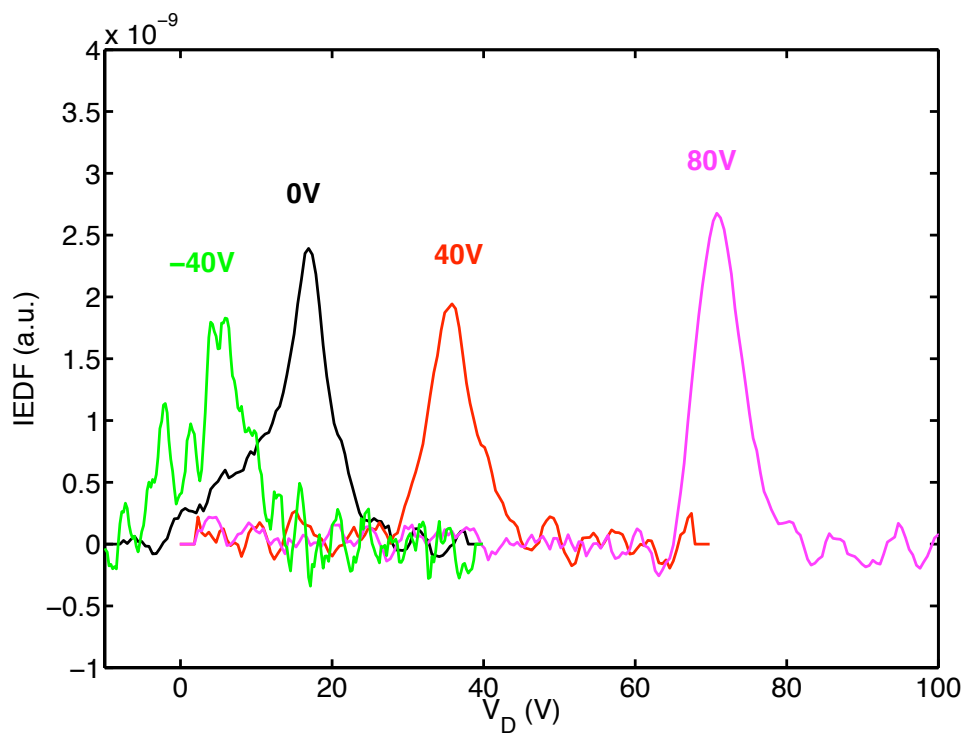

FIG. 10: IEDFs for positive ions in Argon obtained with configuration B for various endplates biases from $-40 \mathrm{~V}$ to $+80 \mathrm{~V}$ ( $V_{e p}$ indicated on each IEDF).

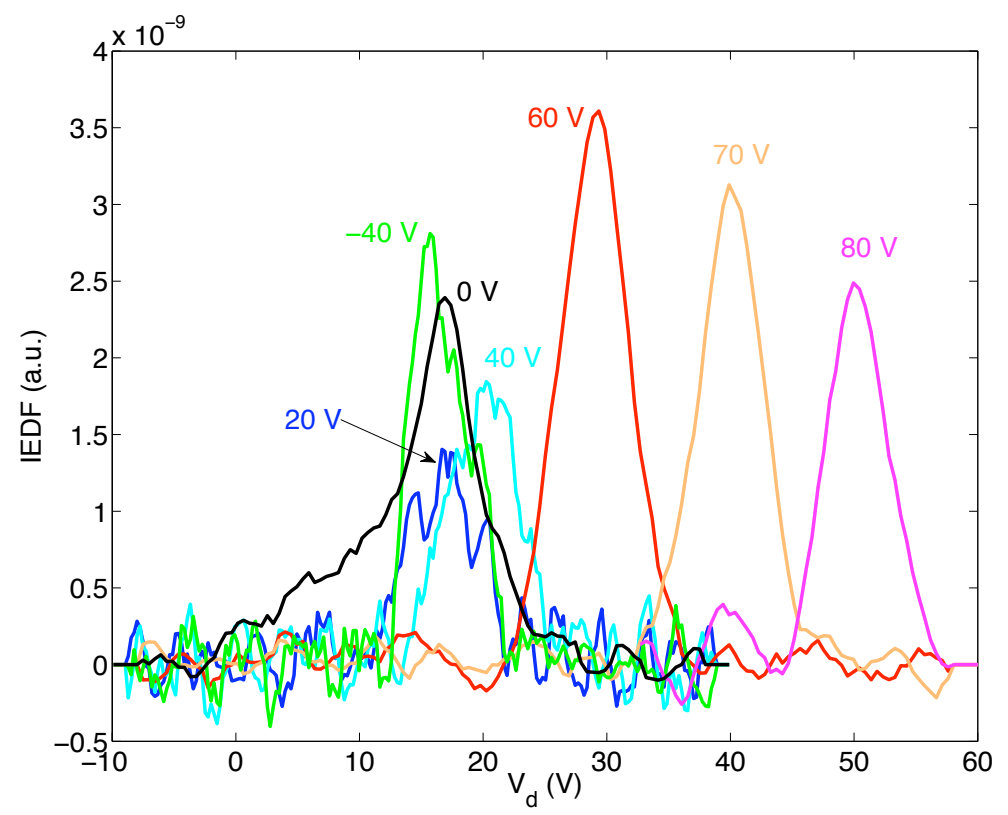

FIG. 11: IEDFs for positive ions in Argon obtained with configuration $\mathrm{C}$ for various endplates biases from $-40 \mathrm{~V}$ to $+80 \mathrm{~V}$ ( $V_{g}$ indicated on each IEDF). 


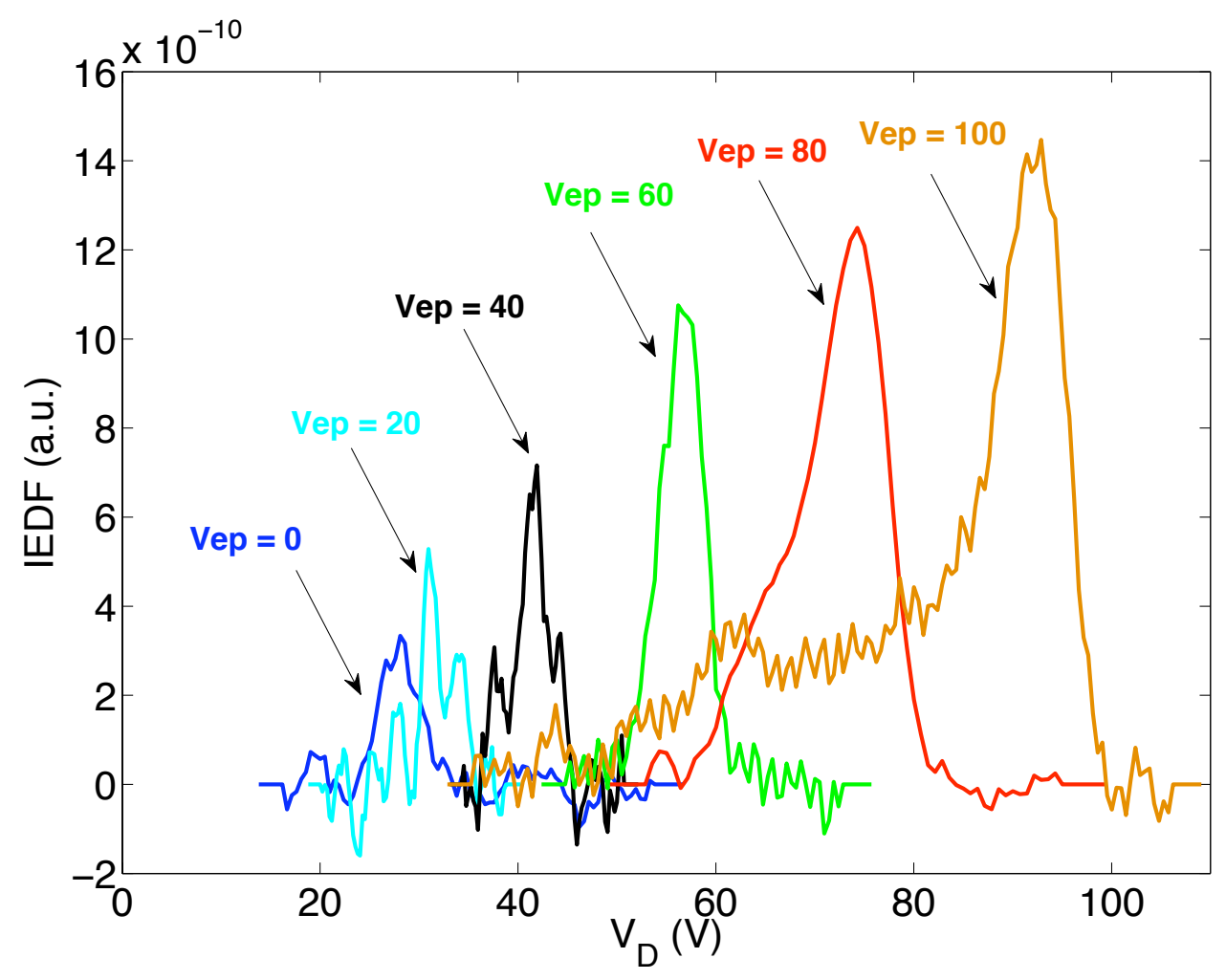

FIG. 12: IEDFs for positive ions in $\mathrm{SF}_{6}$ obtained with configuration $\mathrm{D}$, for various endplates biases from $0 \mathrm{~V}$ to $100 \mathrm{~V}$ ( $V_{e p}$ indicated on each IEDF). 


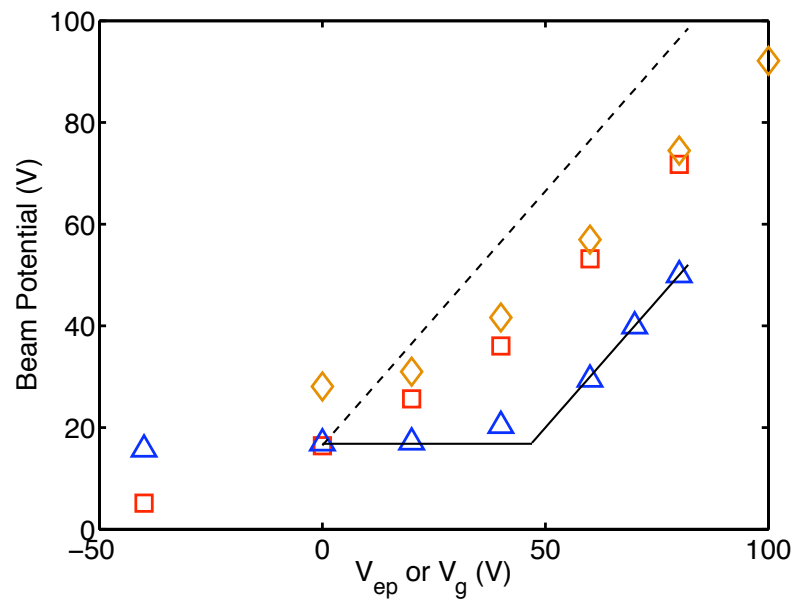

FIG. 13: Beam potential $V_{b}$ as a function of applied bias to either endplates or grid. Red squares obtained from figure 9 (config. B), blue triangles obtained from figure 10 (config. C) and orange diamonds obtained from figure 11 (config. D). The dashed line shows the expected variation of the $V_{b}$, and the solid line shows eq. 4 .

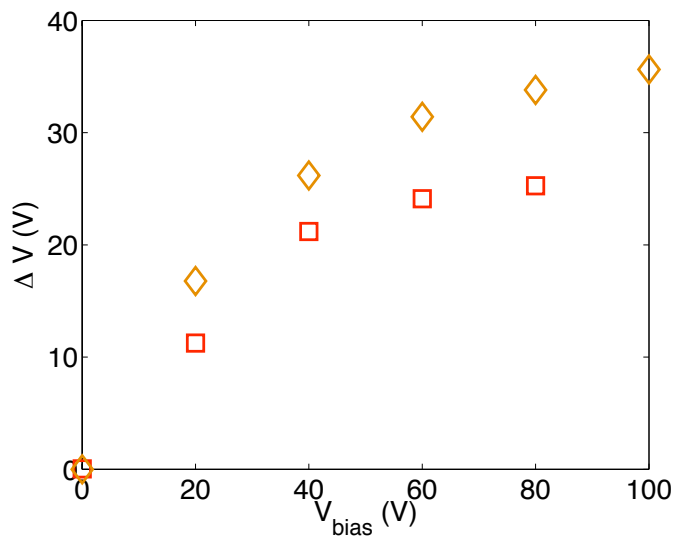

FIG. 14: Loss term $\Delta V$ as a function $V_{e p}$. Red squares corresponds to configuration B (argon) and orange triangles for configuration $\mathrm{D}\left(\mathrm{SF}_{6}\right)$. 